\title{
Collective effects in microscopic transport models
}

\author{
Carsten Greiner ${ }^{\mathrm{a}}$ \\ anstitut für Theoretische Physik I, Universität Giessen, D-35392 Giessen, Germany
}

We give a reminder on the major inputs of microscopic hadronic transport models and on the physics aims when describing various aspects of relativistic heavy ion collisions at SPS energies. We then first stress that the situation of particle ratios being reproduced by a statistical description does not necessarily mean a clear hint for the existence of a fully isotropic momentum distribution at hadrochemical freeze-out. Second, a short discussion on the status of strangeness production is given. Third we demonstrate the importance of a new collective mechanism for producing (strange) antibaryons within a hadronic description, which guarantees sufficiently fast chemical equilibration.

\section{PHILOSOPHY OF HADRONIC TRANSPORT MODELS}

Microscopic hadronic transport models have guided in detail the understanding and interpretation of experimental results of the heavy ion programs at Bevalac, SIS, AGS and SPS energies. For these reasons one can say that this has been the golden age of those models. Competing approaches are the (R)QMD model(s), the URQMD model, the HSD model, the RBUU model(s) and the QGSM model, all including the subsequent microscopic interactions of the hadrons as the common collective feature underlying the dynamics.

These models do provide a complete space-time picture (including momentum space) of a violent heavy ion reaction. They can describe the buildt up of collective effects like flow observables, the production of all kinds of particles, like strangeness and the production of charmonium states, and also electromagnetic probes. The underlying dynamics is described by a phenomenological reaction network of various hadronic Boltzmann processes. As the basic input are vacuum cross sections for the various elastic and inelastic hadronic reaction channels, semiclassical transport approaches do have a profound and solid foundation: relativistic kinetic theory (being, in principle, superior to any thermal or hydrodynamical description) and known vacuum physics.

However, caution enters into the description when cross sections are asked which can not be measured experimentally. Besides the dynamics of all kinds of hadronic resonances and their implementations this is, in particular, true for the modelling of the highly energetic inelastic binary hadronic reactions (by eg string excitation and fragmentation) and their collective incorporation into space-time dynamics. This is the situation where the transport description has a character of a model, as the basic input for the very initial stage of the reaction relies on collective, nonperturbative and non-equilibrium QCD, which can only be mimiced by phenomenological concepts. One has to describe a regime which 
is dominated by soft processes, and which might well also be the situation encountered still at RHIC energies.

In this respect it is clear that transport approaches can not really implement any (pre-) quark gluon plasma (QGP) state of deconfined matter. One can turn this major deficiency into a positive perspective: One can look for observables to see if they show a significant dependence on physics incorporated by the description of the very initial stage of the reaction: Is there any new physics necessary?! In this respect with the transport description at hand one can also implement possible in-medium modifications of particle properties during the course of the reaction and hence test for experimental obervation possibilities.

Momentum equilibration ('thermalization') and chemical equilibration of the various particle species can thoroughly be tested [ 1, 2]. On the other hand, by the success of applying 'thermal' or statistical models (eg [ 3]) to describe the final hadronic yield, it became quite popular to belief that the applicability of such approaches also proves for the existence of a state of almost complete thermal equilibrium. We will stress in section 2 that this does not necessarily mean a clear hint for the existence of a fully isotropic momentum distribution at hadrochemical freeze-out. Therefore the studies of equilibration by hadronic transport models are necessary for a detailed understanding of the reaction dynamics. Second, we will give a brief discussion in section 3 on the status of strangeness production within the models, also refering to an ongoing analysis. Strangeness production had been raised as one of the potential QGP signatures, and here especially rare antihyperons as the important messenger of the initial phase. Refering to the later, section 1 is devoted to a new collective mechanism for producing (strange) antibaryons within a hadronic description [ 4]. So far, the description of the (strange) antibaryon production within standard hadronic transport schemes in comparison to data had faced notorious difficulties. As the new idea has been presented already at the last Quark Matter conference, novel numerical calculations for a dynamical setup are reported.

\section{STATISTICAL MODEL WITH ANISOTROPIC MOMENTUM DISTRIBUTIONS}

Besides the microscopic transport approaches global, statistical analyses as complimentary theoretical descriptions of the final hadronic yield have been explored in detail over the last years (see eg [ 3]). The 'thermal' model description of a noninteracting hadronic resonance gas works astonishingly well to reproduce the various hadronic abundancies by fixing only two intensive parameters, the temperature $T$ and baryochemical potential $\mu_{B}$. From the many analyses one is generally tempted to conclude that at the point where the picture of chemical composition (or 'freeze-out') does apply, a complete thermal state has been established with locally having isotropic distributions in momentum space. On the other hand transport models do not really support the idea that a complete local momentum equilibration can be achieved even at the later stages of the reaction [ [1, 2].

The resolution to this apparent conflict is to relax the constraint of assuming fully isotropic, statistical distributions for comparison to hadron abundancies, i.e. of assuming complete thermal equilibrium [5]. At the onset of the heavy ion reaction the local momentum distribution of the hadronic particles is far from being isotropic. Such a mis- 

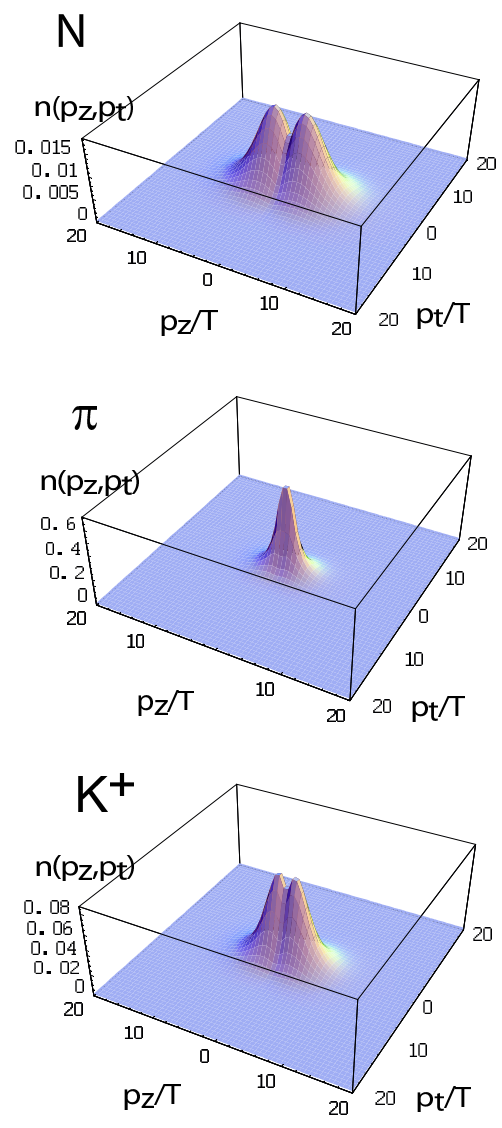

$\mathrm{v}=0.4$
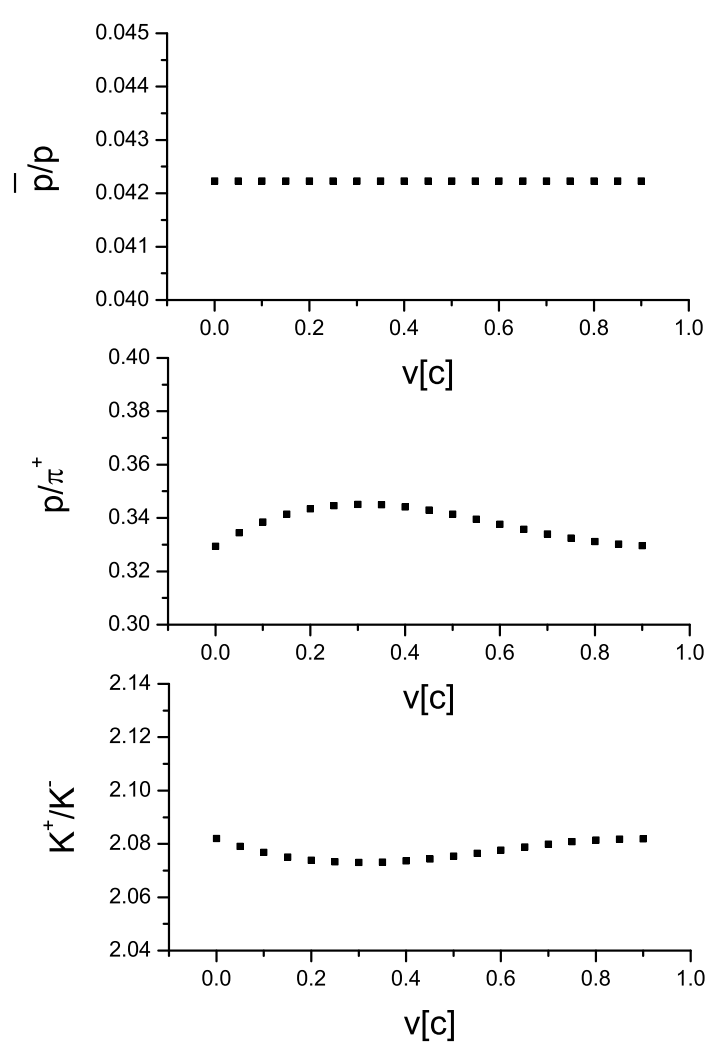

Figure 1. Generalized statistical phase Figure 2. (Weak) Dependence of typical space distribution for $v=0.4$. Temperature parameter and chemical potentials are taken as those extracted for $v=0$ being typical for $\mathrm{Pb}+\mathrm{Pb}$ collisions. particle ratios varying the velocity parameter. Temperature parameter and chemical potentials are taken as those extracted for $v=0$.

match should relax due to collisions among the particles, though the system might never actually become locally completely isotropic [ [1, 2]. To allow for momentum anisotropy within a statistical concept, one has to start from the statistical density operator

$\rho_{G K}=\frac{1}{\Omega} \exp (-\beta(H-\mu N-v O))$,

where $O$ defines the adequate one-particle operator for momentum anisotropy and $v$ is the conjugated intensive quantity [ [6]. For stationarity, the 'von Neumann-equation' $\frac{d \rho}{d t}=\{H, \rho\}=0$ is fullfilled, if $O$ commutes with the (effective) Hamiltonian. If one defines the momentum anisotropy $O$ as the difference between the mean value of the momenta with a positive and negative longitudinal $(z)$ component, respectively, i.e. $\hat{O}(\hat{p})=\sum_{p}\left|p_{z}\right| \hat{a}_{p}^{+} \hat{a}_{p}$, the requirement for stationarity is fulfilled when employing effective 
Hamiltonians of mean-field type or a simple quasi-free Hamiltonian of a hadron resonace gas. The incorporation of binary collisions, of course, will destroy stationarity. Assuming a noninteracting hadron (Hagedorn-like) resonance gas, as typically employed in all analyses, and minimizing the grand canonical potential $\Omega$ leads to the generalized momentum distribution

$\rho_{i}=\frac{g_{i}}{(2 \pi)^{3}} \int d^{3} p \frac{1}{\exp \left(\beta \gamma\left(E_{i}-v\left|p_{z}\right|\right)-\beta \mu_{i}\right) \pm 1}$,

with $\gamma=\left(1-v^{2}\right)^{-1 / 2}$. The intensive parameter $v$ can be interpreted as the relative velocity between projectile and target. Fig. [1 illustrates examples for the case of nucleons, pions and kaons with the intensive parameter $v=0.4 \mathrm{c}$.

In Fig. 2 now the actual (non-)dependence of exemplaric particle ratios on varying the velocity parameter is depicted. The temperature parameter and chemical potentials are taken as those extracted for $v=0$ (i.e. being compatibel with those of [ 3]). The largest deviation, about $6 \%$, one finds for the $p / \pi^{+}$ratio (for the situation of a $\mathrm{Pb}-\mathrm{Pb}$ collisions at SPS). Hence the quality of the fits is not really changed when employing any of these generalized distributions. The same situation of encountering only a very weak dependence is met for an analysis concerning AGS or RHIC data [ 5].

The presented results lead to the conclusion, that the succesful description of particle ratios within a standard statistical model does not give a proof for the stringent existence of local isotropic momentum distribution at hadrochemical freeze-out, but is merely an assumption. The achievement of momentum equilibration can only be judged by considering hadronic transport models in comparison to data.

\section{REMARKS ON STRANGENESS PRODUCTION}

There have been a couple of detailed studies to understand strangeness production for AGS and SPS energies within a specified microscopic model (for reviews see [ 7, 8, 9]). To be specific, in [ 10] the properties of $K^{+}, K^{-}$and $\Lambda$ particles in nuclear reactions from SIS to CERN-SPS energies have been investigated within the HSD model. Here also available $\mathrm{p}+\mathrm{A}$ data has been confronted. The outcome for the excitation function of the $K^{+}$-mesons, relative to the $\pi^{+}$-yield is summarized in fig. 3. After the primary string fragmentation of intrinsic p-p-collisions the hadronic fireball starts with a $K^{+} / \pi^{+}$ ratio still far below chemical equilibrium with $\approx 6-8 \%$ at AGS to SPS energies before the hadronic rescattering starts. Secondary (meson-baryon) and ternary (meson-meson) induced string-like interactions do then contribute significantly to additional strange particle production, particular for reactions at SPS energies. Via these channels about the same number of strange and anti-strange quarks is produced as in the primary $\mathrm{p}+\mathrm{p}$ collisions. This then can explain the relative enhancement compared to $\mathrm{p}+\mathrm{p}$ (see fig. 3). It is valid to say that the so called strangeness enhancement at SPS energies does not require deconfinement.

The major amount of produced strangeness (kaons, antikaons and $\Lambda \mathrm{s}$ ) at SPS-energies can be understood in terms of early and still energetic, non-equilibrium hadronic interactions. Afterwards, more or less no further strangeness is being produced in any of the microscopic hadronic transport simulations. Putting it differently, when the momenta of the nucleons have sufficiently degraded and the system has to some extent thermalized, 
the timescale for production of strange particles via the considered kinetic reactions becomes much too large. Fig. As summarizes this statement: The chemical saturation of the kaons has been investigated microcanonically for a static box and is found to be larger than $40 \mathrm{fm} / \mathrm{c}$ for all equilibrium energy densities up to $2-3 \mathrm{GeV} / \mathrm{fm}^{3}$ and thus exceeds considerably the lifetime of the fireball [2].

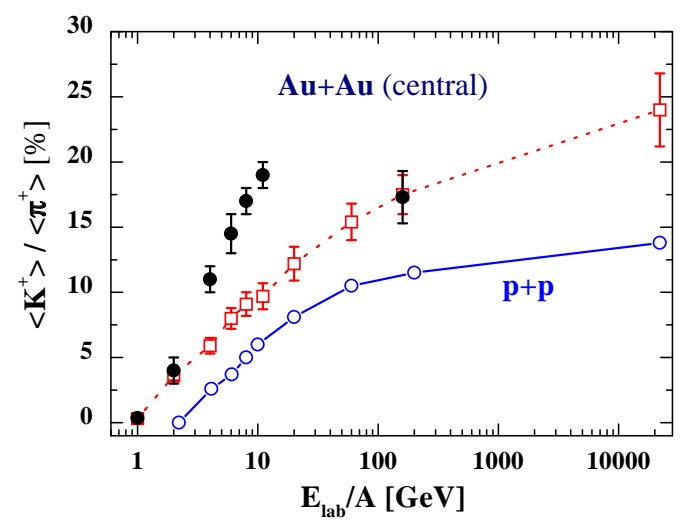

Figure 3. Excitation function for $K^{+} / \pi^{+}$around midrapidity for central $\mathrm{Au}+\mathrm{Au}$ reactions (open squares) from SIS to RHIC energies obtained within the HSD model in comparison to experimental data and elementary $\mathrm{p}+\mathrm{p}$ collisions (open circles).

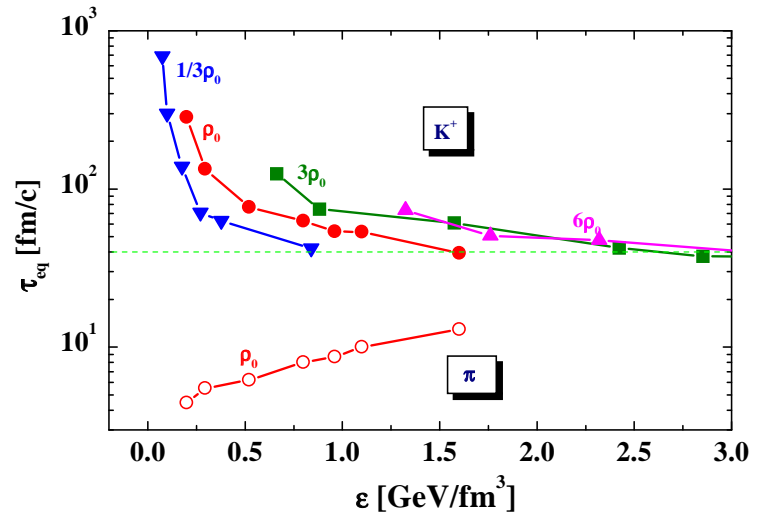

Figure 4. Chemical equilibration time versus energy density for $\pi$ and $K^{+}$mesons at various different baryon densities [2].

Focussing on lower bombarding energies, a smooth and continously smaller $K^{+} / \pi^{+}$is seen in the simulation (compare Fig. 3), which clearly is not in line with the behaviour seen at AGS energies or at lower SPS energies! Strangeness production is underestimated by $\approx$ $30 \%$ compared to explicit kaon data [10] wheras the pion population is in addition slightly overestimated. The outcome of three microscopic transport simulations has been reported in [ 8]. URQMD calculations do find agreement for the lower AGS energies, but otherwise appreciably do underestimate the ratio. However, again when looking on the pion yield and the kaon yield seperately, one realizes that the difference to the experimental rapidity distributions is still sizeable, but not as dramatic [11]. Here, at SPS energies and for highest AGS energies the pions are slightly overestimated, whereas the kaons are slightly underestimated, the two and opposite tendencies then are enlarged when showing only the ratio.

One conclusion thus is that showing the $K / \pi$-ratio can distort the results, which actually should be better compared seperately. The second and important conclusion is that one really has to understand the physics reasons for the minor, but still significant difference in the results (like pion and kaon distributions) among the various transport approaches, as the underlying principle philosophy is the same. It could be, on the one hand, that 
the primary and violent stage of the reaction is modelled differently, which then might lead to noticeable differences in some observables. As for the AGS energies and the lower SPS energies the baryon densities achieved are the highest, especially then the baryonic resonances could be of crucial importance and thus could also be a possible source for the reported differences. Hence, on the other hand, it might be that also the treatment and thus the understanding of the later hadronic stage of the reaction is of significant relevance. A detailed comparison is presently carried out by Bratkovskaya and Weber [ 11.

\section{4. (STRANGE) ANTIBARYON PRODUCTION}

In particular because of high production thresholds in binary hadronic reaction channels antihyperons had been advocated as the appropriate QGP messengers. Indeed, a satisfactory picture of nearly chemically saturated populations of antihyperons has been demonstrated over the last years with the $\mathrm{Pb}+\mathrm{Pb}$ experiments NA49 and WA97 at CERN-SPS [3]. On the other hand the theoretical description of the antibaryon production within hadronic transport schemes in comparison also to earlier data for light systems faced some severe difficulties. Phenomenological motivated attempts [ 12] to explain a more abundant production of antihyperons had been proposed like the appearance of color ropes, the fusion of strings, the percolation of strings, or the formation of high-dense hadronic clusters. Their purpose is mainly to create (much) more antibaryon in the very early intial stage of the reaction (compared to simple rescaled $\mathrm{p}+\mathrm{p}$ collisions). Still, in most of the transport calculations a dramatic role of subsequent antibaryon annihilation is observed, which, in return, has to be more than counterbalanced by the initial production. The situation seems even more paradox with respect to the fact that the chemical description by thermal models works indeed astonishingly well for the antihyperons and being, of course, completely nondependent on the (large) magnitude of the annihilation cross section. For all of this reasons the theoretical and dynamical understanding of the production of (strange) antibaryons has remained a delicate and challenging task [ 7].

Amazingly, it was noticed only recently that a correct incorporation of the baryonic annihilation channels had actually not been done consistently in the simulations [ 13, 47. A sufficiently fast redistributions of strange and light quarks into (strange) baryonantibaryon pairs should be achieved by multi-mesonic fusion-type reactions of the type

$n_{1} \pi+n_{2} K \leftrightarrow \bar{Y}+p$

occuring in a moderately dense hadronic system [4]. Naively one thinks that the probability of multiple pions and kaons to come close in space is very low and therefore irrelevant. However, (at least) these special kind of multi-hadronic reactions have to be present because of the fundamental principle of detailed balance.

In the following we present rate calculations for a dynamical setup of an expanding (and nearly equilibrated) hadronic resonance gas. Ideally the rate equations do correspond to a coarse grained, effective description of microscopic hadronic transport. We will turn to brief discussion of how to implement such reactions within a microscopic, local transport at the end of this section. For the expansion either a linear profile $R(t)=R_{0}+v_{\text {lin }}\left(t-t_{0}\right)$ or an accelerating profile $R(t)=R_{0}+v_{0}\left(t-t_{0}\right)+0.5 a_{0}\left(t-t_{0}\right)^{2}$ is employed for the effective 


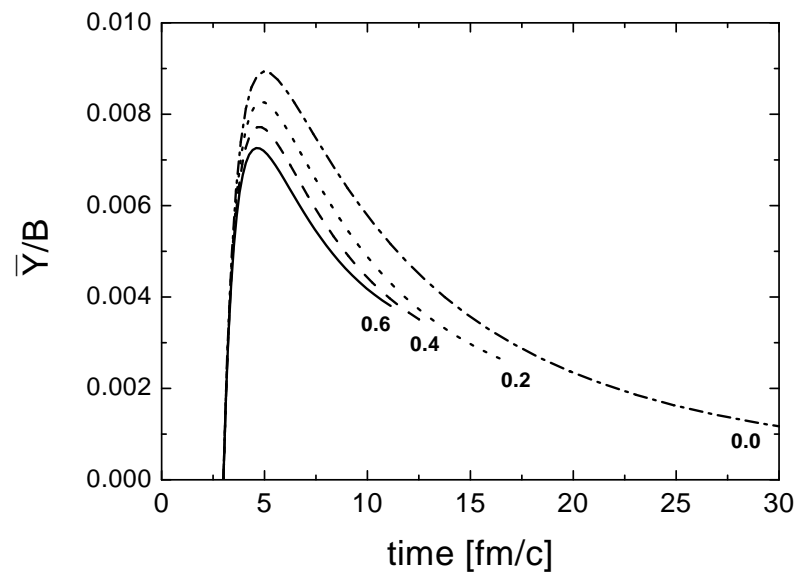

Figure 5. The anti- $\Lambda$ to baryon number ratio $N_{\bar{\Lambda}} / N_{B}(t)$ as a function of time for various velocity parameters $v_{l i n}$ for the transverse expansion. The entropy per baryon is taken as $S / A=30, t_{0}=3 \mathrm{fm} / \mathrm{c}$ and $T_{0}=190 \mathrm{MeV}$.

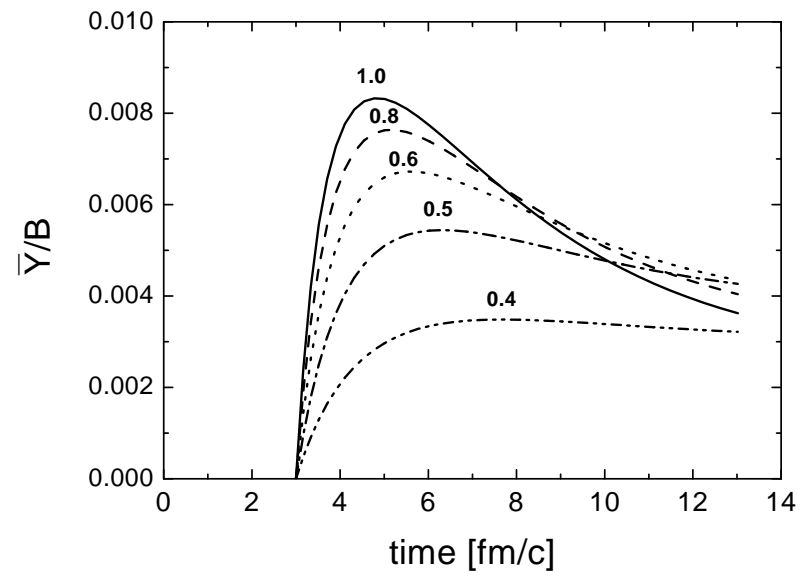

Figure 6 . The anti- $\Lambda$ to baryon number ratio $N_{\bar{\Lambda}} / N_{B}(t)$ as a function of time for various implemented annihiation cross section $\sigma_{e f f} \equiv \lambda \sigma_{0}$. The entropy per baryon is taken as $S / A=30, t_{0}=3 \mathrm{fm} / \mathrm{c}$ and $T_{0}=190 \mathrm{MeV}$.

volume expansion $V(t) \sim t * R^{2}(t)$. The evolution of the hadronic gas is taken to be an isentropic expansion being specified via the entropy per baryon ratio $S / A$. Temperature and baryon density are then evaluated as a function of time, once an initial temperature and time has been chosen (for more details see the last reference of [ [4]).

In Fig. 5 the number of $\bar{\Lambda}$ s (normalized to the conserved net baryon number) as a function of time is depicted. As a minimal assumption the initial abundancy of antihyperons is set to zero in all of the following figures (and actually will not depend on the initial value (!)). The entropy per baryon is chosen as $S / A=30$ being a typical value to global (' $\left.4 \pi^{\prime}\right)$ SPS results. The parameter $v_{\text {lin }}$ is varied to simulate slow or fast expansion of the late hadronic fireball. The general characteristics is that first the antihyperons are dramatically being populated, and then in the very late expansion some more are still being annihilated, depending on how fast the expansion goes. A rapid expansion gives a higher yield, which can increase the final yield by a factor of 2 to 3 . Still, the typical expansion behaviour obtained from simulations or extracted from the analysis of transverse momentum slopes of pions and protons is that at the late stages the transverse expansion velocity should be about $0.5 \mathrm{c}$.

In Fig. [ the number of $\bar{\Lambda}$ s as a function of time is depicted, where now the annihilation cross section employed is varied by a constant factor, i.e. $\sigma_{\text {eff }} \equiv \lambda \sigma_{0}$ [ [4]. For the volume expansion an appropriate transverse radius accelerating in time is now employed. The results are rather robust against a variation by a factor of 2 in the cross section. Typically (for $\lambda=1$ ) about more than 5 times in number of antihyperons are created during the evolution compared to the final number freezing out, thus reflecting the fast back ('annihilation') and forth ('creation') processes at work dictated by detailed balance. 
The mass law action enforces the hyperons to buildt up and then maintain chemical equilibrium for a certain time, until at moderately low particle densities the multi-mesonic fusion processes are getting ineffective due to the rapid dilution.

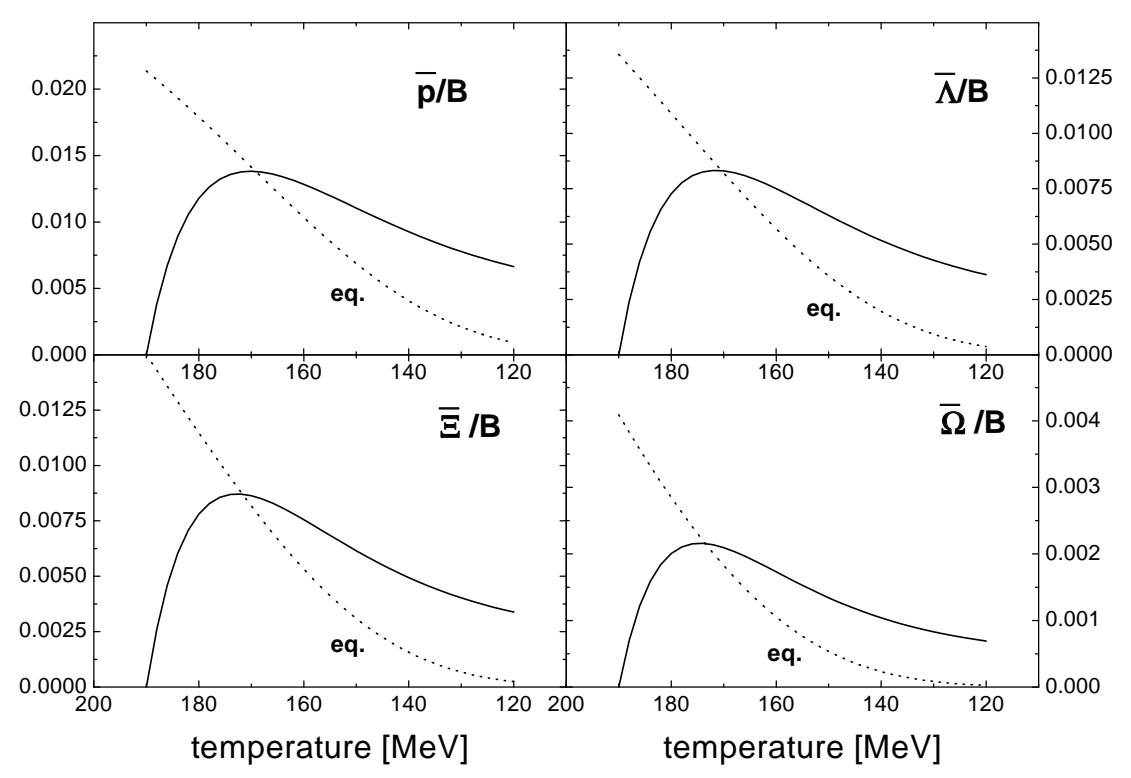

Figure 7. The antihyperon to baryon number ratio $N_{\bar{Y}} / N_{B}(T)$ and $N_{\bar{Y}}^{e q .} / N_{B}(T)$ (dotted line) as a function of the decreasing temperature. Parameters are the same as in Fig. 6.

In Fig. 7 the number of antihyperons of each specie and the direct anti-protons (not coming from any anti-baryon resonance) are now shown as a function of the decreasing temperature $T(t)$ of the hadronic system. For comparison the instantaneous equilibrium abundancy $N_{\bar{Y}}^{e q .}\left(T(t), \mu_{B}(t), \mu_{s}(t)\right) / N_{B}$ is also given. As noted, after a fast initial population, the individual yields of the antihyperons do overshoot their respective equilibrium number and then do finally saturate at some slightly smaller value. Moreover, one notices that the yields effectively do saturate at a number which can be compared to an equivalent equilibrium number at a temperature parameter around $T_{\text {eff }} \approx 150-160$ $\mathrm{MeV}$, being close to the ones obtained within the various thermal analyses [ 3]. To be more quantitative in comparison with data, one has at midrapidity the WA97 results $\bar{\Xi}^{+} / \bar{\Lambda} \equiv \bar{\Xi}^{+} /\left(\bar{\Lambda}+\bar{\Sigma}^{0}\right) \approx 0.188 \pm 0.016$ and $\bar{\Omega}^{+} / \bar{\Xi}^{+}=0.281 \pm 0.053$ [ 14]. Assuming that $N_{\bar{\Lambda}} \approx N_{\bar{\Sigma}^{0}}$ and $N_{\overline{\bar{\Xi}^{+}}} \approx N_{\bar{\Xi}^{0}}$ we have (from Fig. (7) $\bar{\Xi}^{+} /\left(\bar{\Lambda}+\bar{\Sigma}^{0}\right) \rightarrow 1 / 4 * \bar{\Xi} / \bar{\Lambda} \approx 0.22$ and $\bar{\Omega}^{+} / \bar{\Xi}^{+} \rightarrow 2 * \bar{\Omega} / \bar{\Xi} \approx 0.35$, both values being in good agreement to the data.

In Fig. B the number of anti- $\Lambda \mathrm{s}$ as a function of time is given for various entropy per baryon ratios. One notices that the final value in the yield significantly depends on the entropy content, or, in other words, on the baryochemical potential. We note that the absolute yield on $\bar{\Lambda}$ s at midrapidity from WA97 and NA49 can best be reproduced by employing an entropy to baryon ratio $S / A \approx 40$ : From WA97 we have about $1.8 \pm 0.2[3]$ 


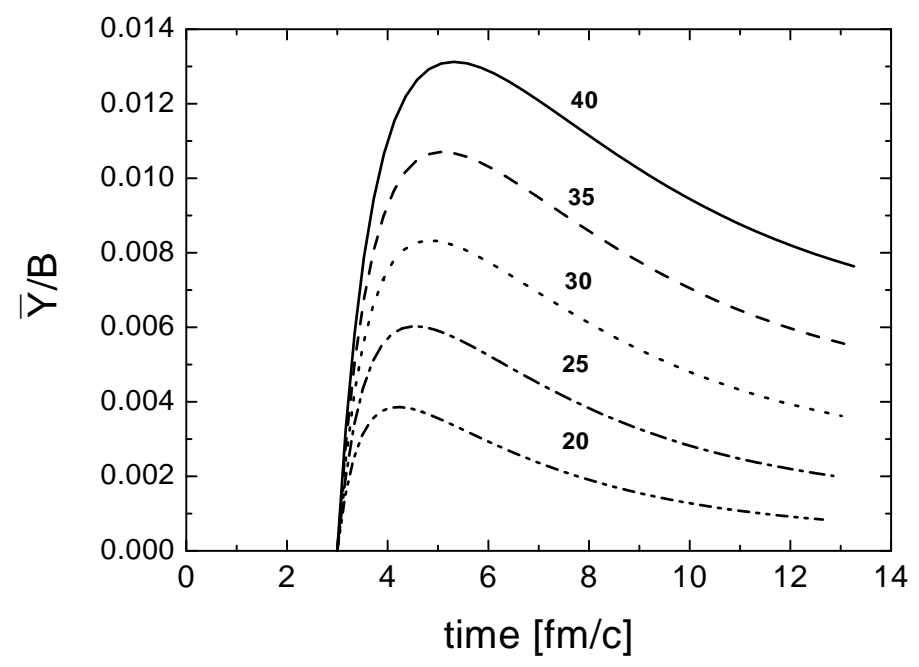

Figure 8. $N_{\bar{\Lambda}} / N_{B}(t)$ as a function of time for various entropy content described via the entropy per baryon ratio $(S / A=20-40)$. Other parameters are as in Fig. 6.

and from NA49 about 1.5 [15] anti-Lambdas (and $\bar{\Sigma}^{0} \mathrm{~s}$ ) in one unit of rapidity. Assuming again that $N_{\bar{\Lambda}} \approx N_{\bar{\Sigma}^{0}}$ and taking for the net baryon rapidity distribution $d N_{B} / d y \approx 80$ one has $N_{\bar{\Lambda}} / N_{B} \approx(1.5 / 2) / 80 \approx 0.0094$, which would be roughly the outcome for $S / A$ being slightly larger than 40 (compare Fig. 7). Indeed, at midrapidity one qualitatively expects a higher entropy content due to the larger pion to baryon ratio as compared to full ' $4 \pi$ ' data over all rapidities. At this point it will also be very interesting to compare our semi-quantitative calculations with the new results from NA49 on the $\bar{\Lambda}$-yield at lower SPS energies of $80 \mathrm{AGeV}$ and $40 \mathrm{AGeV}$ with lower entropy contents, respectively [15].

To summarize, multi-mesonic production of antihyperons is a consequence of detailed balance and, as the annihilation rate is large, it is by far the most dominant source in a hadronic gas. It clearly demonstrates the importance of hadronic multi-particle channels, occuring frequently enough in a (moderately) dense hadronic environment in order to populate and chemically saturate the rare antibaryons. In order to be more competitive for a direct comparison with detailed experimental findings (like $A_{\text {part }}$ dependence), new strategies have to be developed to describe for such multi-particle interactions within present day transport codes. A significant first step forward was very recently been pursued by Cassing, where the concept of rate calculations in subdivided, rather local space-time cells has been adopted. The annihilation cross section is modelled by threebody vector meson decay. First results concerning the production of anti-protons at AGS and SPS energies are quite impressive [16]. (At SPS energies the $\bar{p}$-yield was calculated to be nearly a factor of two below the published, yet rather old data. A new experimental analysis, but still not presented in public, shows that the actual experimental $\bar{p}$-yield will be reduced by nearly a factor of two [17]. This would mark the importance of this specific calculation.) Another strategy for implementing the backreaction could be to incorporate microscopically the concept of two meson doorway states [18] and their sequential decay by standard binary scattering processes. 


\section{Acknowledgments}

The study presented in section 2 was done together with Björn Schenke. The author is also indebted to E. Bratkovskaya and $\mathrm{H}$. Weber for illuminating discussions. This work was supported by the Bundesministerium für Bildung und Forschung, by the Deutsche Forschungs Gemeinschaft and by the Gesellschaft für Schwerionenforschung.

\section{REFERENCES}

1. M. Belkacem et al, PRC 58, 1727 (1998); L. Bravina et al, Phys. Lett. B 434, 379 (1998); PRC 60, 024904 (1999); J. Sollfrank, U. Heinz, H. Sorge and N. Xu, PRC 59, 1637 (1999).

2. E. Bratkovskaya, W. Cassing, C. Greiner, M. Effenberger, U. Mosel and A. Sibirtsev, Nucl. Phys. A 674, 249 (2000).

3. P. Braun-Munzinger, I. Heppe and J. Stachel, Phys. Lett. B 465, 1 (1999); F. Becattini, J. Cleymans, A. Keränen, E. Suhonen and K. Redlich, PRC 64, 024901 (2001).

4. C. Greiner and S. Leupold, J. Phys. G 27, L95 (2001); C. Greiner, Nucl. Phys. A 698, 591 (2002); and arXiv:nucl-th/0208080.

5. B. Schenke and C. Greiner, in preparation.

6. L. Neise, GSI report 90-24 (1990), unpublished.

7. H. Sorge, Nucl. Phys. A 630, 522c (1998).

8. S.A. Bass, J. Phys. G 28, 1543 (2002).

9. C. Greiner, J. Phys. G 28, 1631 (2002).

10. J. Geiss, W. Cassing and C. Greiner, Nucl. Phys. A 644, 107 (1998).

11. H. Weber, E. Bratkovskaya and H. Stöcker, arXiv:nucl-th/0205030; H. Weber, E. Bratkovskaya et al, in preparation.

12. H. Sorge et al, Phys. Lett. B 289, 6 (1992); H. Sorge, Z. Phys. C 67, 479 (1995); Phys. Rev. C 52, 3291 (1995); K. Werner and J. Aichelin, Phys. Lett. B 300, 158 (1993) and Phys. Lett. B 308, 372 (1993); N. Armesto, M.A. Braun, E.G. Ferreiro and C. Pajares, Phys. Lett. B 344, 301 (1995); E.G. Ferreiro and C. Pajares, Z. Phys. C 73, 309 (1997); M. Bleicher et al, Phys. Lett. B 485, 133 (2000).

13. R. Rapp and E. Shuryak, Phys. Rev. Lett. 86, 2980 (2001).

14. R. Caliandro for the WA97 collabortion, J. Phys. G 25, 171 (1999).

15. A. Mischke, these contributions.

16. W. Cassing, Nucl. Phys. A 700, 618 (2002).

17. P. Seyboth, private communications.

18. J. Vandermeulen, ZPC 37, 563 (1988). 